\title{
Poétiques sur le pas de porte : de la magopinaciophilie comme terrain littéraire
}

\section{Ninon Chavoz}

\section{OpenEdition}

\section{Journals}

Édition électronique

URL : https://journals.openedition.org/coma/3871

DOI : 10.4000/coma.3871

ISSN : 2275-1742

\section{Éditeur}

Institut des textes \& manuscrits modernes (ITEM)

\section{Référence électronique}

Ninon Chavoz, "Poétiques sur le pas de porte : de la magopinaciophilie comme terrain littéraire », Continents manuscrits [En ligne], 12 | 2019, mis en ligne le 08 mars 2019, consulté le 12 janvier 2023. URL : http://journals.openedition.org/coma/3871 ; DOI : https://doi.org/10.4000/coma.3871

Ce document a été généré automatiquement le 12 janvier 2023.

\section{(c) $(1) \&$}

Creative Commons - Attribution - Pas d'Utilisation Commerciale - Pas de Modification 4.0 International - CC BY-NC-ND 4.0

https://creativecommons.org/licenses/by-nc-nd/4.0/ 


\title{
Poétiques sur le pas de porte : de la magopinaciophilie comme terrain littéraire
}

\author{
Ninon Chavoz
}

C'est mieux quand tu es plus sage, plus lent, que
tu retrouves ta majesté, que tu traces chaque
lettre comme si tu la faisais naître, comme si tu la
composais tel un peintre ou un prêtre, un
marabout merveilleux. Car tu es un marabout
merveilleux ${ }^{1}$.

1 Puisées à pleines mains au fond des boîtes aux lettres, les merveilles des marabouts ne sont pas de celles qu' "invente» une chasse au trésor. À Paris comme dans de nombreuses métropoles françaises, ils font désormais figure d'incontournables gardiens des seuils, répandant leur omniprésent «magic marketing» dans les espaces liminaires - couloirs, bouches de métro et halls d'immeubles. Situés dans une zone transitionnelle entre l'Europe et l'Afrique, leurs prospectus surchargés de texte nourrissent en Occident un émerveillement pour partie exotisant, quand ils ne vont pas jusqu'à attiser une secrète fièvre collectionneuse : à tout le moins transforment-ils la boîte aux lettres qui les accueille en un terreau voire en un terrain littéraire - fût-il modestement réduit à l'espace de quelques centimètres carrés.

2 L'investigation de ces cartes de visite relève en premier lieu d'une anthropologie attentive aux points de contact et autres «branchements ${ }^{2}$ » interculturels. Dans l'entreprise "d'ethnologie à rebours " qu'il consacre aux «marabouts de Paris", Massaër Diallo souligne ainsi la spécificité d'un regard porté d'abord sur les «ressemblances, inattendues ou cachées » plutôt que sur des «valeurs et traditions spécifiques» jugées par trop "évanescentes dans ce qui pourrait fonder leur singularité ou leur exclusivité $»^{3}$. Un tel postulat pourrait être analysé à la lumière des « lectures croisées » récemment proposées par Souleymane Bachir Diagne et Jean-Loup Amselle. Définissant un universalisme qu'il qualifie de «matriciel», fondé sur le 
"postulat de principes communs à plusieurs cultures", ce dernier développe en effet un exemple tout à la fois spirituel et matérialiste, finalement placé sous le signe complexe du « fétiche »:

On peut d'ailleurs apporter à l'appui de ce point de vue contre-intuitif l'existence de nombreuses pratiques se situant à cheval sur l'Afrique et l'Europe, comme le recours à la voyance par certains chefs d'État - Valéry Giscard d'Estaing et François Mitterrand par exemple -, ou bien encore la religion de la consommation qui se traduit par la croissance exponentielle de la quantité de déchets et qui peut être comparée, toutes choses égales par ailleurs, aux sacrifices effectués en Afrique sur les fétiches ${ }^{4}$.

3 Le lien avec les marabouts s'impose ici doublement, soit qu'on le situe dans l'élan spiritiste des présidents français, exploré, comme on le verra plus loin, dans un roman de Thierry Pfister, soit qu'on entende le retrouver dans la débauche consumériste des "déchets", auxquels se voient souvent réduits les confettis publicitaires dont regorgent les boîtes aux lettres.

\section{De la magopinaciophilie aux contre-littératures}

4 La textualité exponentielle de ces prospectus ne saurait cependant être considérée uniquement comme une rançon de l'éphémère. Tout au contraire, les cartes de visites de marabouts ont suscité de longue date une passion conservatrice dont la manifestation la plus flagrante est sans doute l'« encyclopédie magopinaciophile » constituée en ligne depuis 2005, et qui se targue aujourd'hui de rassembler près de 2000 flyers classés et répertoriés ${ }^{5}$ (fig. 1). Ce néologisme de formation récente, imputé à un certain Jean Claveret, entend répondre à la gageure de " trouver un équivalent grec à une chose pas du tout grecque - $[. .]:. 1^{\circ}$ un prospectus ; $2^{\circ}$ un marabout ${ }^{6} »$. Pour désigner celui-ci, c'est le terme de magos qui est retenu, précisément parce qu'il qualifie souvent les sorciers mèdes et perses. Pour le prospectus publicitaire, le lexicographe averti recourt au pinakion, qui désigne les planchettes utilisées par les juges pour énoncer leur verdict. Le terme ainsi obtenu est agréablement ronflant, érigeant l'humble collectionneur de ces petites cartes, fréquemment livrées au caniveau, au rang d'érudit à la spécialisation étroite, voire grandiloquente : "magopinaciophilie, ce n'est plus qu'un simple passe-temps, c'est majestueux, c'est grandiose !7 ». 
Figure 1 : La « Galerie des flyers » sur le site web de la magopinaciophilie

\section{La Galerie des flyers}

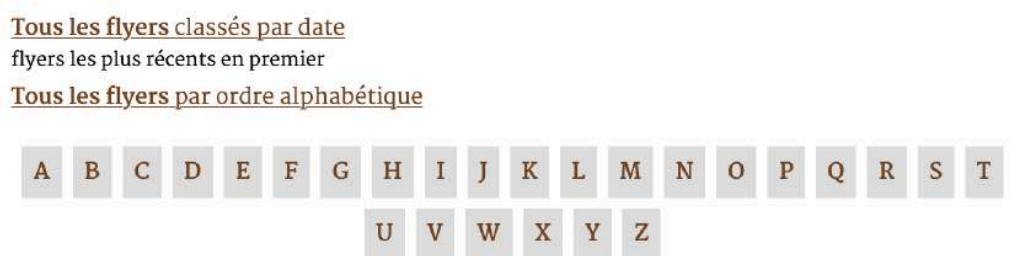

Liste des flyers de marabouts, en partant du plus récent (date d'insertion dans la base) : 1882 flyers trouvés.

\begin{tabular}{|l|l|l|l|l|l|l|l|l|l|l|}
\hline CISSE & NSAMA MADY & \multicolumn{2}{|c|}{ MOHAMED } & \multicolumn{2}{c|}{ Keita Souleymane } & ALIOU & ABA \\
\hline GASSAMA & TAMADI & SOUGANDI & KAN & SOUARÉ & SOARES & DIOUARA \\
\hline MAÏGA & MOUC & KANDJOURA & DIALLO & DIBA & DAOUDA & BILLALY \\
\hline BANA & BAFODE & BAMBO & BABA & BABA & MOHANLIM AZIZ & AMADOU \\
\hline ALY & ALAIN & ALIMOU & ALI & HASSAN & ANSOU KEITA & \\
\hline GOULOU EXERCICE & FANGAMA & TOURE & KANDJOU & DACOSTA \\
\hline TOURÉ MOHAMED & SYLLA & SOUARE & SEYNI & SEYNI & \multicolumn{2}{|l|}{ SEKHOUBA } \\
\hline
\end{tabular}

5 Où ranger pourtant cette magopinaciophilie, toute majestueuse qu'elle soit, dès lors qu'on entend lui trouver une place dans le panthéon des sciences humaines? Autant qu'à une géographie urbaine ou à une anthropologie des branchements, il semble que cette ramification du savoir s'apparente bel et bien à l'analyse du discours : outre les recommandations adressées aux collectionneurs, le portail de la magopinaciophilie comporte ainsi un répertoire des tropes les plus employés par les marabouts, proposant une terminologie idiosyncrasique de "figures imposées" parmi lesquelles on citera «l'analogie canine» (fig. 2), le "recyclage littéraire» (illustré entre autres par l'insensible transition de vos problèmes inquiétants ou tracassants, prêté au Professeur Beba, à vos problèmes inquiétants ou fracassants chez le professeur Aydala), «l'énumération hétéroclite", «la prolifération pictogrammatique » (fig. 3) et la très paradoxale "spécialisation généraliste », dont l'expression la plus limpide réside dans la qualification large de "spécialiste en la matière " (fig. 4). La magopinaciophilie serait donc d'abord une étude de style, sensible aux récurrences, aux choix illustratifs et aux effets rhétoriques. À ce titre, elle pourrait être rapprochée de l'étude des contrelittératures dont Bernard Mouralis identifiait dès 1975 les multiples supports, irréductibles à la raison graphique du livre ${ }^{8}$. Au rang de ces textes souvent considérés comme "non-littéraires", il citait entre autres les encyclopédies, les "prospectus en tous genres ", les " petites annonces », les «formulations publicitaires » et l' "activité scripturaire underground»- autant de catégories dans lesquelles il serait loisible de classer les volatiles cartes de marabouts. Selon Bernard Mouralis, la contre-littérature ne se définit cependant pas exclusivement par son "statut» de minoration: en se refusant à reprendre la qualification de "paralittérature ", il entend en effet souligner la capacité disruptive de textes « qui, par leur seule présence menacent déjà l'équilibre du champ littéraire ${ }^{9}$ " et contribuent à en redessiner les lignes. Dans le roman de Patrick Grainville, la consultation du guérisseur Auguste Traoré vise ainsi à guérir un 
écrivain de la crampe qui interdit les mouvements de sa main et l'empêche de poursuivre son œuvre: on ne saurait mieux dire que le marabout prolixe a pour charge la cure d'une paralysie des mots.

Figure 2 : Deux exemples de flyers utilisant l'analogie canine

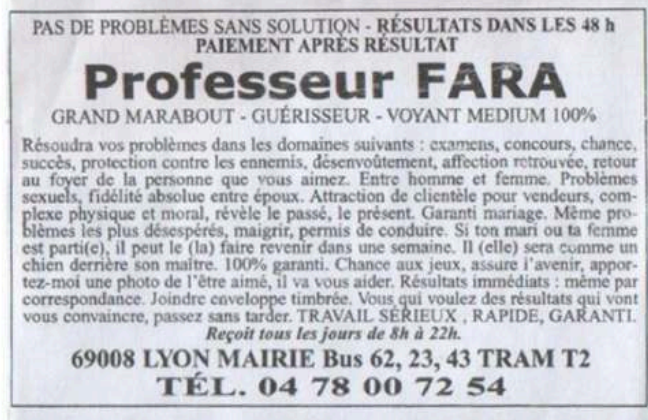

\section{GRAND CHEIKH MAMADOU VOYANT}

EFFICACITEEN $4 B$ - PAIEMENT APRES SATISFACTION

II vous aide dans tous los domaines Spécialiste des

travaux d'amour, retour définitif de la personn

aimée, si votre amour vous a quitté(e), il (elle) va

courir derrière vous comme un chien derriere son maitre, sexualití, chance, travail, protection contre les ennemis, réussite en affaires, commerce, examens, concours, provoque, attire, renforce les

sentiments, désenvoutement, etc..
Reçoit sur rendez vous, ou par correspondance

Tèl 01.55.79.14.74 Port. 06.20.08.68.84

24 RUE LAGHOUAT 75018 PARIS

Métro Chấteau Rouge 


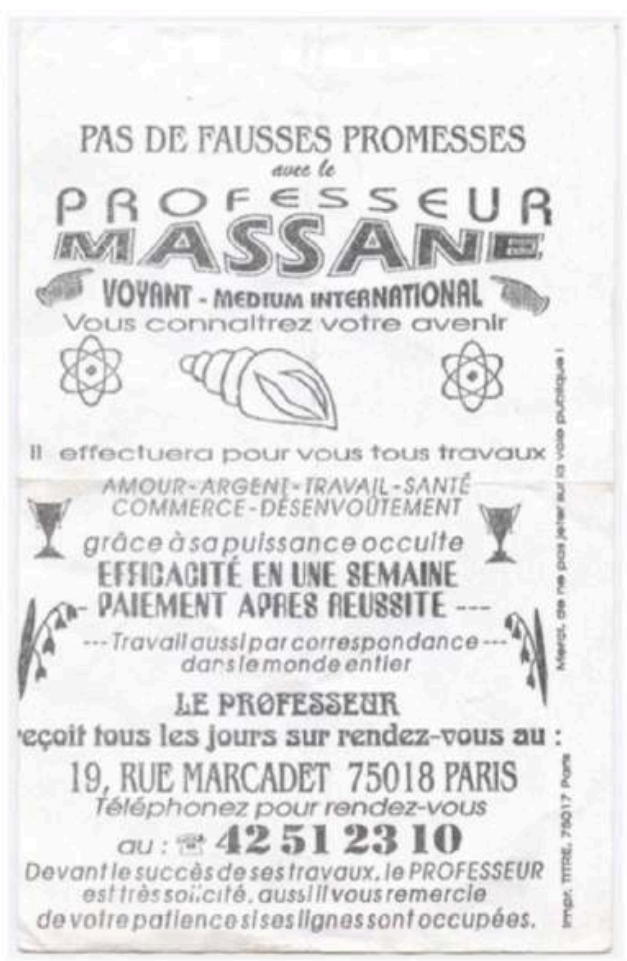

Figure 4 : Exemple de « spécialisation généraliste »

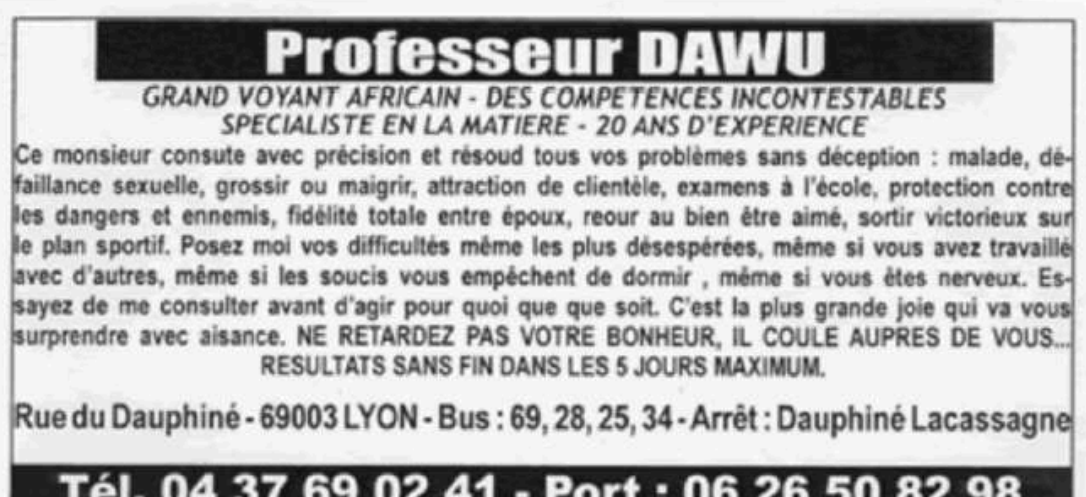

6 Bien que l'encyclopédie magopinaciophile se pare de la légèreté d'une plaisanterie potache, parfois entachée d'un exotisme bon marché, nous souhaiterions donc prendre cette collection au sérieux en la considérant non pas seulement comme une forme spécifique de literacy ${ }^{10}$ mais comme le ferment d'une contre-littérature. De fait, si le marabout parisien reste un personnage discret dans les romans des écrivains francophones contemporains, l'étude du plus textuel d'entre ses attributs invite à inventer un terrain littéraire pour retracer les circulations interculturelles de fragments susceptibles de se muer en autant de bribes poétiques. Parler du marabout par l'entremise de ses cartes de visite revient dès lors à envisager une esthétique des seuils 
et à examiner les conditions d'une rencontre manquée entre ces manieurs de mots et de merveilles et l'écrivain francophone contemporain.

\section{Anthropologie urbaine et littérature : les chemins croisés du marabout et du sapeur}

7 La situation des marabouts parisiens a fait l'objet de plusieurs études approfondies, dont il est frappant de constater qu'elles s'adossent systématiquement à une préoccupation littéraire au sens large du terme. Le texte que Blaise N'Djehoya consacre en 1984 aux «Français vus par les Africains" voisine ainsi avec une étude «d'ethnologie à rebours » menée par Massaër Diallo au sujet de la clientèle des marabouts ${ }^{11}$. Le " regard noir " qu'entend porter cette étude passe par l'investigation d'une pratique qui, tout en portant au cœur des sociétés occidentales le coin d'une pensée magique, constitue également un mode d'occupation littéraire de l'espace urbain, où fleurissent les cartes promotionnelles et où sévissent les rabatteurs. Plus récemment, l'anthropologue Liliane Kuczynski a consacré un ouvrage détaillé aux origines et aux réseaux des marabouts parisiens en soulignant d'emblée le rapport étroit qui s'établit entre l'activité maraboutique et le maniement du verbe ${ }^{12}$. Tout en rappelant l'importance de l'écriture dans une pratique fondée sur la connaissance du Coran, elle remarque la spécificité d'un langage devenu proprement parisien :

Il fallait donc éclairer ce vocabulaire et en même temps se conformer au terrain : prendre acte du langage employé à Paris, de cet usage large des mots ${ }^{13}$.

8 La particularité de la situation des marabouts immigrés tient selon elle à un double phénomène d'émancipation vis-à-vis de l'héritage dynastique africain et d'insertion dans le réseau préconstitué de l'occultisme français. En se livrant à plusieurs études de cas, l'anthropologue montre comment l'immigration parisienne autorise un décloisonnement de la spécialisation maraboutique, traditionnellement réservée à certaines familles, en même temps qu'elle conduit les marabouts à élargir le spectre de pratiques déjà éclectiques. Ces derniers reprennent ainsi à leur compte des traditions ésotériques locales - la cabbale juive, le tarot ou le magnétisme - dont ils s'approprient les modes de diffusion et le vocabulaire. On mentionnera à titre d'exemple Ibrahim Bobo qui se présente comme "marabout", "guérisseur ", "voyant" et, dans une tradition littéraire toute française, «moraliste » (fig. 5). Le Professeur Lamine Mahaba se proclame quant à lui "spécialiste des travaux occultes", versé à la fois dans la "géomencie » et dans "l'asrare", tout en illustrant son prospectus d'un profil de licorne (fig. 6). «L'usage large des mots » et des images serait le pendant lexical de cet éclectisme : perméable aux savoirs indigènes ainsi qu'à leurs lexiques spécifiques, le marabout se livre volontiers à un «bricolage » textuel autant que spirituel ${ }^{14}$. Ces traits distinctifs incitent l'anthropologue à considérer le sujet de son étude comme un " personnage protéiforme » et complexe $\mathrm{e}^{15}$, irréductible à une assignation univoque. 


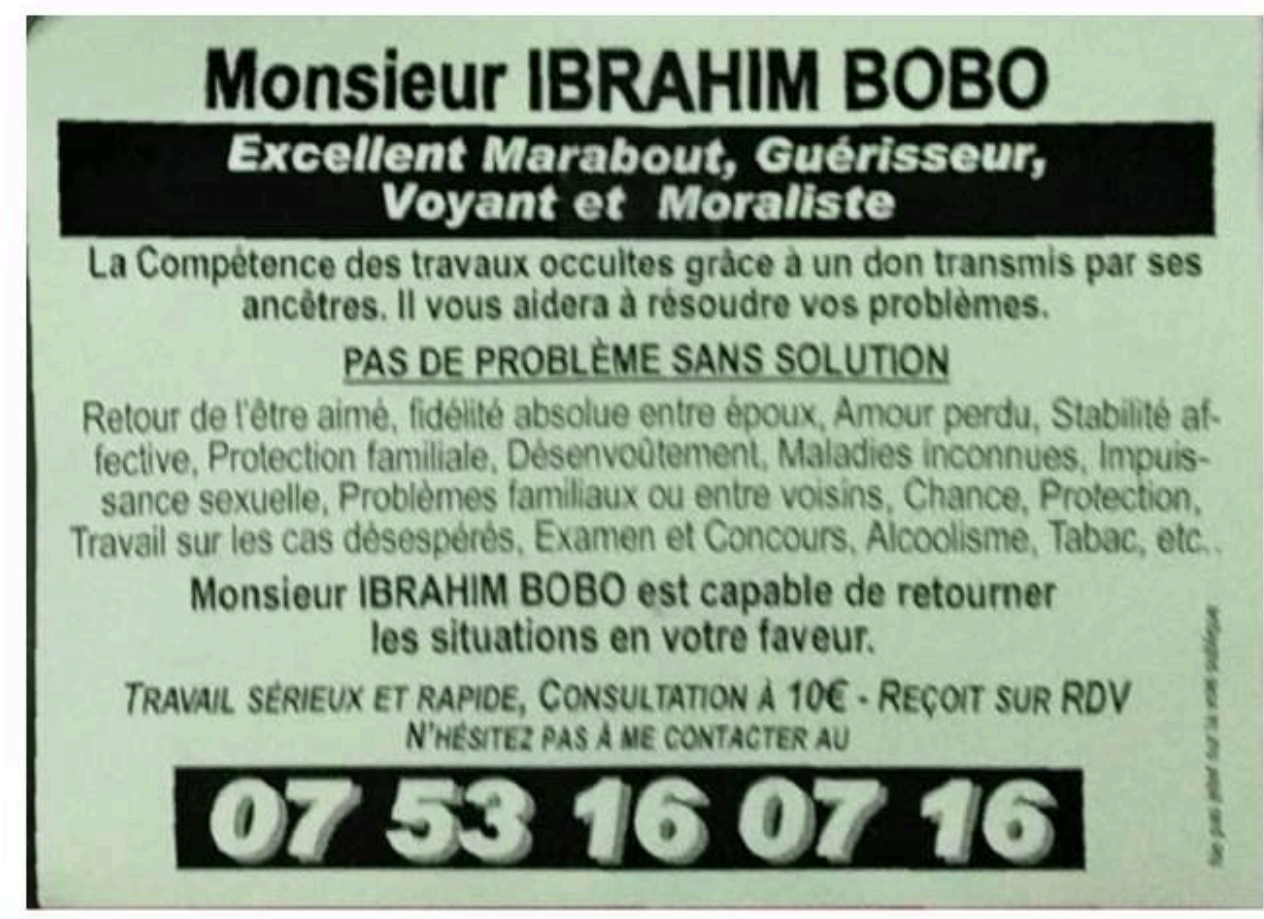

Figure 6 : Exemple de flyer d'un marabout se disant versé dans la géomencie et dans l'asrare

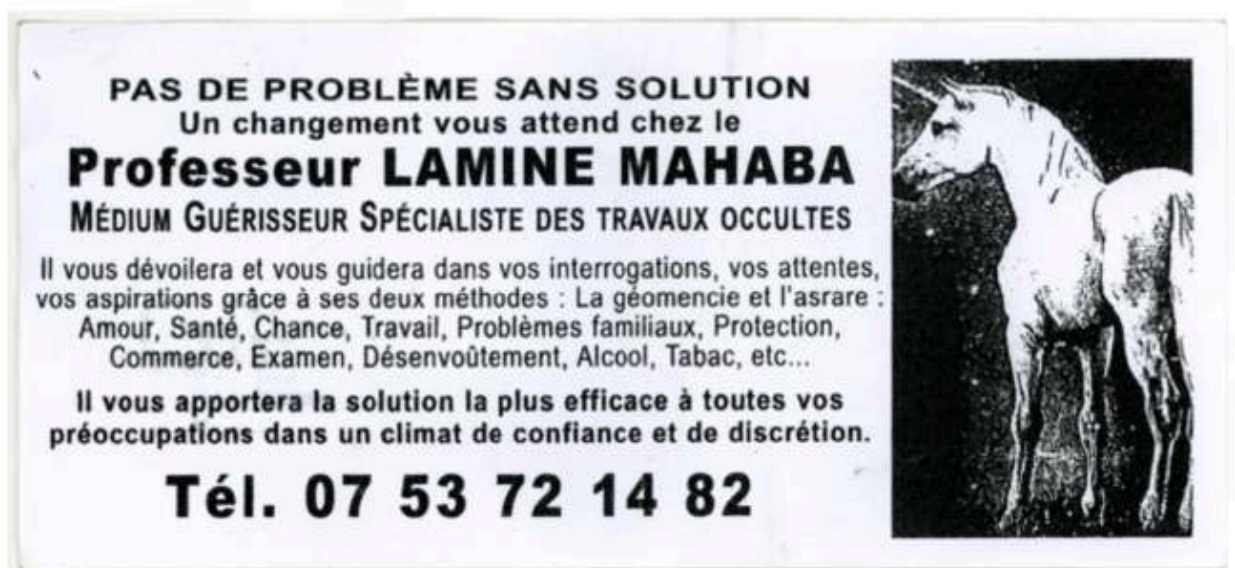

Prendre cette qualification au pied de la lettre et traiter le marabout comme un «personnage » à part entière implique assurément de penser la transition du terrain anthropologique au terrain littéraire. Un tel glissement pourrait être rapproché de celui qu'a connu la figure du sapeur, qui franchit rapidement les bornes de l'essai sociologique ${ }^{16}$ pour se couler dans la matière romanesque: de même que le sapeur incarne par ses atours chamarrés un versant de l'Aventure congolaise, le marabout parisien, porteur d'attributs et d'accessoires éminemment reconnaissables, apparaît comme une personnification remarquable de la migration ouest-africaine, et il pourrait à ce titre occuper en littérature une place aussi prépondérante que son rival congolais $^{17}$. En 1986, le film Black Mic-Mac réalisé par Thomas Gilou faisait d'ailleurs voisiner ces deux figures stéréotypées de l'immigration africaine, présentées comme les 
pôles opposés du faste et de la spiritualité : fasciné par les sapeurs dont il observait avec ravissement le défilé, Lemmy, interprété par Isaach de Bankolé, se faisait passer pour le saint homme qu'il avait rencontré dans l'avion ${ }^{18}$ (fig. 7). L'entrée en littérature du marabout paraîtrait en outre d'autant plus légitime que, comme le sapeur exhibant dans sa danse les griffes de marques vestimentaires, il pourrait, par la pratique de l'intertextualité qu'implique la composition de ses cartes de visite, constituer non seulement un personnage romanesque et une matrice poétique, mais aussi un modèle pour l'écrivain postcolonial.

Figure 7 : Personnage de marabout dans une scène du film Black Mic-Mac

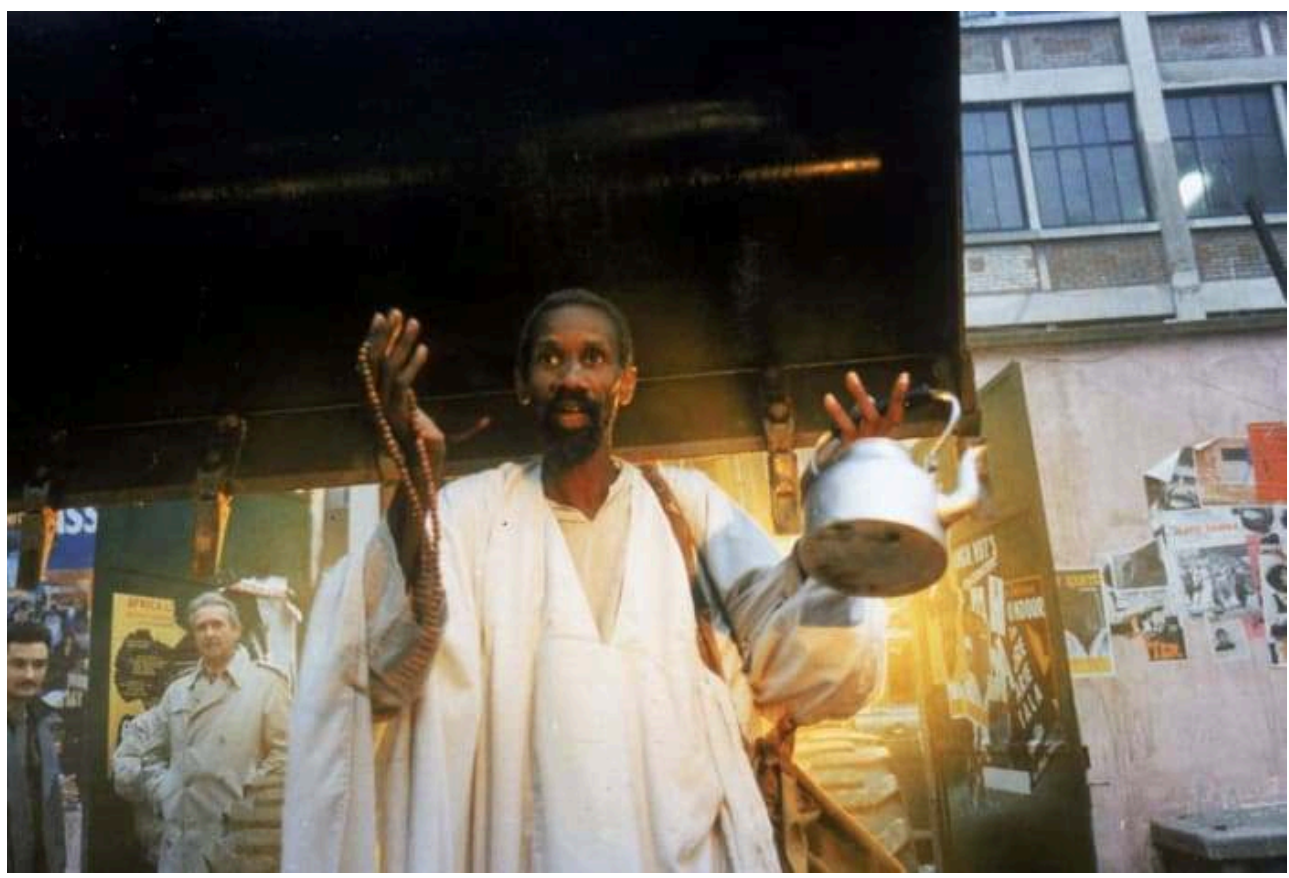

Pourtant, lorsqu'elle évoque l'entrée en littérature du marabout, Liliane Kuczynski souligne avant tout sa présence dans un roman politique de Thierry Pfister ${ }^{19}$ et dans un récit introspectif de Laure Adler ${ }^{20}$ - sans évoquer jamais sa présence dans des textes d'auteurs qu'on pourrait rapprocher du courant dit de «la migritude ${ }^{21}$ ». Force est en effet de constater dans ce domaine une relative absence du marabout, aussi négligé que le sapeur se voit mis en valeur. Là où le second a récemment été introduit en grande pompe au Collège de France $^{22}$, les apparitions littéraires du premier demeurent étrangement rares et fragmentaires, cantonnées à un chapitre ou à un paragraphe isolé, dans lesquels le personnage fait office de figurant discret et souvent peu recommandable.

\section{Apparitions liminales : le marabout au seuil du récit}

11 Sans prétendre à l'exhaustivité, nous nous arrêterons pour illustrer ce constat sur deux romans parus en 2000 : Comment cuisiner son mari à l'africaine de la romancière d'origine camerounaise Calixthe Beyala et Place des fêtes, du romancier d'origine togolaise Sami Tchak. Chacun de ces deux récits rédigés à la première personne pourrait être considéré comme un roman de formation, mettant en scène la quête d'un narrateur tourmenté par les angoisses identitaires autant que par les affres amoureux. 
12 Par son seul titre, le roman de Sami Tchak s'inscrit dans la géographie parisienne des marabouts décrite par Liliane Kuczynski. Malgré cet ancrage dans le XIX arrondissement, le personnage du marabout demeure pourtant très marginal, et sa consultation n'est envisagée qu'à titre hypothétique. Il apparaît ainsi lorsque le narrateur cherche à se venger de son professeur de mathématiques, coupable d'avoir terni à force d'allusions grivoises le nom de sa mère :

J'aurais même payé les services d'un marabout sénégalais de Barbès pour le tuer si j'avais eu beaucoup d'argent, parce que le marabout, il était là, planté à la sortie du métro Barbès et il tendait à tout le monde des prospectus sur lesquels il se permettait de s'appeler Docteur Professeur Savant Machin de Kaolack. Il disait qu'il pouvait tout pour tout le monde. C'était courageux ce qu'il faisait, parce que ses petits papiers, quand il les donnait aux gens, eh bien, les gens les lui rejetaient sur le pied et il ne se décourageait pas $^{23}$.

Le marabout devient ici un véritable chiffonnier du verbe, dont les serments grandiloquents, rapidement résumés dans une périphrase hyperbolique, sont voués à finir au caniveau : le personnage confirme à cet égard le constat amer d'une difficulté d'intégration, voire d'une certaine humiliation de l'immigré qu'énonce le héros. Le surgissement de ce marabout mis au rebus coïncide néanmoins avec un tournant décisif dans la vie du jeune homme: l'insulte proférée par le professeur l'incite en effet à acquérir une meilleure connaissance de la sexualité féminine et lui ouvre les portes d'un érotisme qui constitue l'un des fils directeur du roman. Quant à l'enseignant coupable, il connaît un châtiment qui se dispense de tout truchement maraboutique en allant se perdre au cœur des ténèbres africaines.

Dans le bref récit de Calixthe Beyala ${ }^{24}$, l'intervention du marabout marque également l'orée de la métamorphose que connaît la narratrice, soucieuse de séduire son voisin Monsieur Bolobolo. Aïssatou amoureuse se résout à solliciter les conseils d'un initié, se pliant ainsi à l'axiome des «vieillards " restitué au début du sixième chapitre, selon lequel « une Africaine sans marabout est comme un navigateur sans boussole » (p. 43). Expliquant à Aïssatou, en bon «amoureulogue» (fig. 8), qu'elle n'est pas «amoureusable» (p.48) parce qu'elle manque de formes et ne sait pas cuisiner, le marabout la conduit à justifier le titre du récit en se transformant en pourvoyeuse de délices aussi bien érotiques que gastronomiques. À cet égard, il est l'agent d'un véritable retour aux origines - qu'il s'agisse de recouvrer le goût pour des plats aussi exotiques que le "boa en feuilles de bananier" ou de renoncer au modèle de la « Négresse blanche» (p. 21). Accueillie chez le marabout par l'assistante du maître et par les clientes qui l'ont précédée, Aïssatou se voit d'emblée placée dans une situation d'inconfort et de mauvaise foi :

- Je cherche le professeur Gombi, dis-je.

Et j'ajoute précipitamment :

- Pas pour ce que vous pensez.

- Et je pense quoi, selon la très chère?

[...]

Les justifications de ma présence chez un marabout les révulsent. Une Blanche - qui a déjà compris que la magie africaine peut l'aider à obtenir RETOUR DANS LES VINGTQUATRE HEURES DU BIEN-AIMÉ - AFFECTION IMMÉDIATE - FIDÉLITÉ À L'INFINI - SATISFACTION SUR LE CHAMP OU REMBOURSÉ - casse sa bouche de mépris et baisse les yeux. [...] Les Négresses sont regroupées entre elles et maudissent les hommes: "Des coureurs! Des menteurs! Des briseurs de cœurs! » Je suis inquiète dans mon coin. (p. 45-46). 


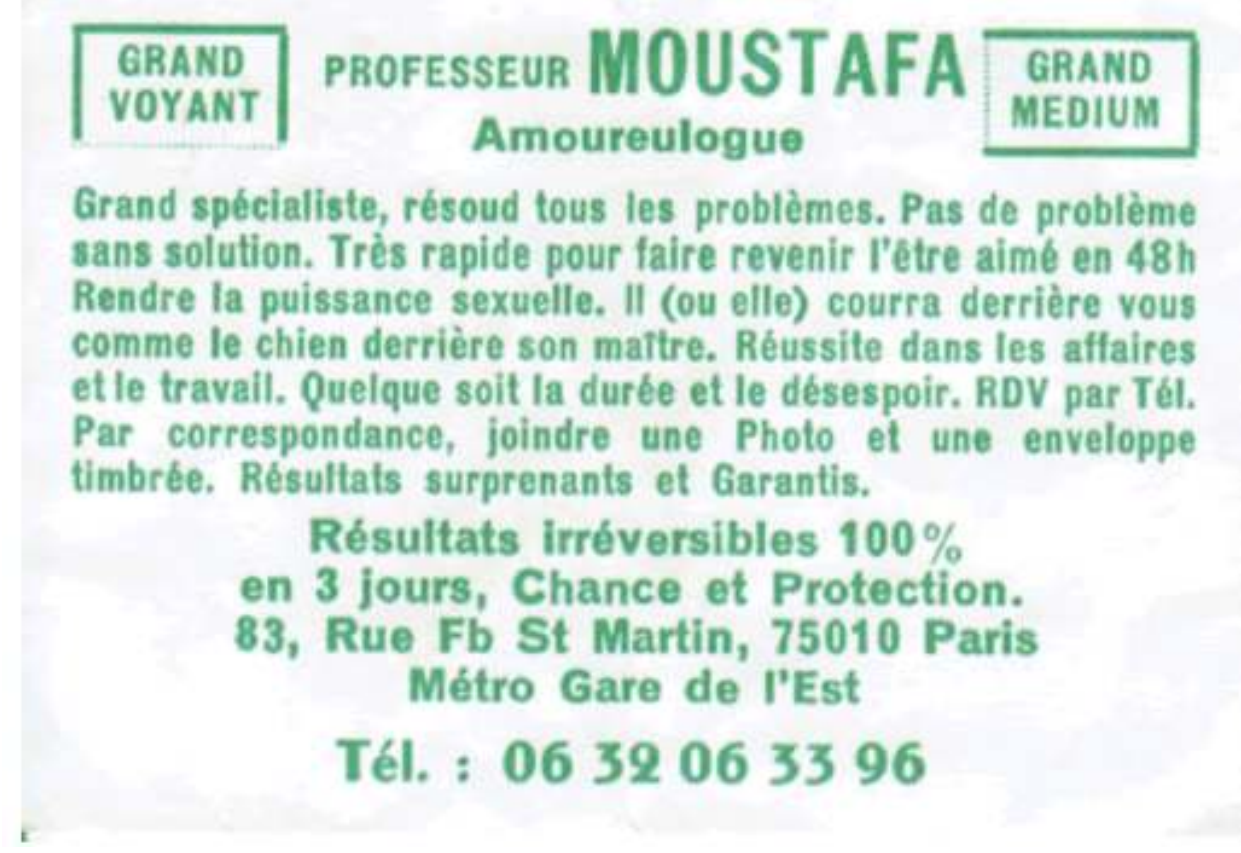

La rencontre avec le professeur Gombi n'a lieu qu'après le passage par ce purgatoire féminin, où la narratrice se trouve confrontée au vertige spéculaire de sa propre identité partagée. Le porteur de promesses disparaît ensuite du roman et ne ressurgit que dans les dernières pages, lorsque la concierge d'Aïssatou vient la consulter pour lui demander l'adresse d'un marabout susceptible de l'aider à reconquérir son époux volage. Loin de se contenter de transmettre les coordonnées du professeur Gombi, Aïssatou reconduit alors l'attitude de l'assistante qui l'avait reçue: après avoir embarrassé la visiteuse en égrenant quelques remarques salaces, elle lui prodigue ellemême ses conseils. D'un pas de porte à l'autre, les cartes ont donc été rebattues: assumant son identité franco-africaine, Aïssatou est passée maîtresse en matières amoureuses, et sait désormais garantir elle-même le célèbre « retour dans les vingtquatre heures du bien-aimé ».

Dans les deux cas, la présence du marabout, quand bien même elle se situe à un tournant du roman de formation et invite à un « retour au pays natal " plus ou moins heureux, demeure donc fugitive : loin d'opérer des prodiges, le personnage ne bénéficie que d'un statut douteux, oscillant entre le mendiant et l'escroc ${ }^{25}$.

\section{Composer en marabout, ou les allers-retours de l'être aimé}

Placer le marabout au seuil d'une Afrique stéréotypée ou le renvoyer à un soupçon de charlatanerie déjà avéré à l'époque coloniale ${ }^{26}$ ne suffit cependant pas à rendre compte de la «complexité » du personnage que pointe l'anthropologue. Bien plus, sa vertu littéraire nous semble résider, ainsi que le suggère le roman de Patrick Grainville, dans un talent textuel de "composition » qui trouve une expression patente dans les cartes de visite maraboutiques. Non content de proposer de plaisants montages photographiques (fig. 9) ainsi que l'adaptation de jeux aussi impérissables que le taquin 
(fig. 10), le portail de la magopinaciophilie, dans son volet «Laboratoire » invite ainsi ses usagers à s'essayer au maniement d'un programme informatique, le «Personal Marabout Generator ", présenté dans des termes rabelaisiens :

Ces flyers ont été analysés afin d'en extraire la substantifique moelle. C'est grâce à cette étude que le PMG produit aujourd'hui des simulations si réalistes. À dire vrai, même les authentiques marabouts peuvent aujourd'hui utiliser le PMG pour générer leurs propres flyers ! ${ }^{27}$

Figure 9 : Exemple de montage photographique : les marabouts à la Maison Blanche

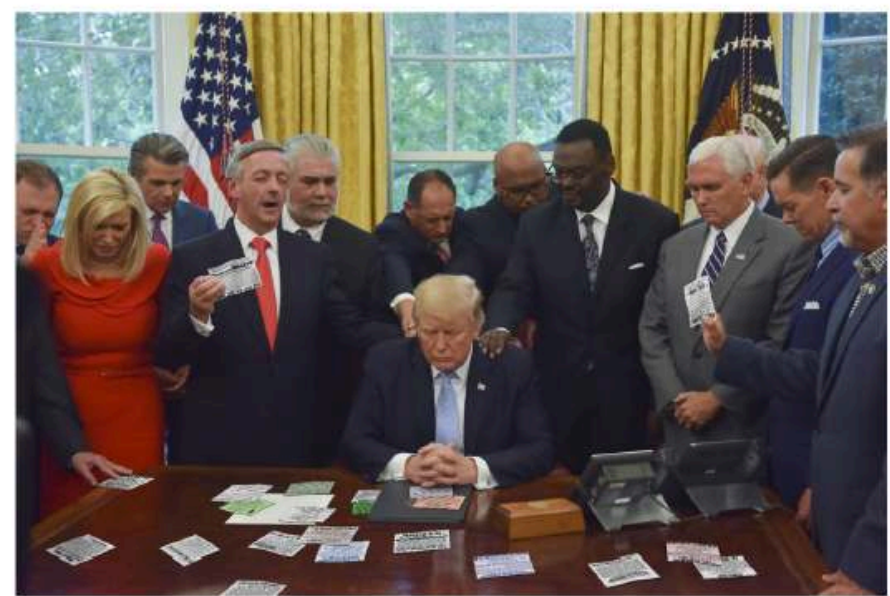

Le document photographique exceptionnel que vous voyez ci-dessus a été "fuité" par un proche collaborateur de Trump, en 2017, au péril de sa vie. Que s'est-il passé ce jour-là, dans les premiers mois de l'administration Trump ? En faisant le point sur sa collection, le Président s'aperçoit qu'un flyer rare de M. DAFFE manque à l'appel. Branle bas de combat immédiat dans le bureau ovale! Tous les conseillers et secrétaires d'état sont immédiatement mis à contribution pour partir à la recherche du précieux exemplaire perdu. L'ambiance est extrêmement tendue. Trump est hors de lui et déverse sa fureur sur son entourage terrorisé - chacun est un coupable potentiel ! Après plusieurs heures de recherches infructueuses, il faut se rendre à l'évidence : aucune trace de M. DAFFE. Trump craque et se met à pleurer à chaudes larmes. Il faudra toute la patience et la gentillesse du staff pour le consoler, certains allant jusqu'à offrir comme consolation de nouveaux flyers fraîchement collectés. Évidemment, la tristesse et la compassion qui se lisent sur les visages sont un peu forcées, mais que ne ferait-on pas pour témoigner son empathie au grand chef ? 
Figure 10 : Page du site web de la magopinaciophilie proposant de jouer au taquin.

\section{Le marabout taquin}

Pouvez-vous reconstituer le flyer?

Le flyer de marabout que vous voyez ci-dessous a été découpé en petits carrés qui ont été mélangés. Cela donne ce qu'on appelle un taquin (en savoir plus...). A vous de le reconstituer, le plus rapidement possible. Il y a plusieurs modèles différents. Vous pouvez jouer avec n'importe quel flyer de la galerie (sur n'importe quelle fiche, cliquer sur "Jouer au taquin avec ce flyer").

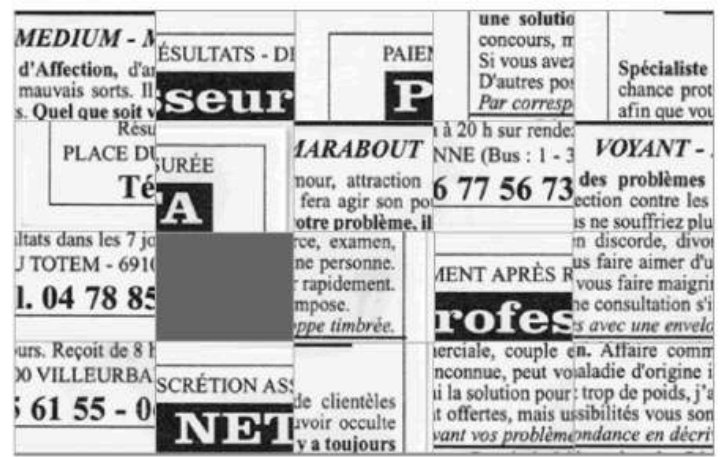

o coup joué

18 Le collectionneur occidental offrirait ainsi aux marabouts « authentiques » un dispositif de promotion automatisé, fondé sur un art de la combinatoire oulipiste, comparable aux Cent mille milliards de poèmes de Raymond Queneau (fig. 11). Pourtant, l'examen attentif des pratiques scripturaires des marabouts permet de porter un regard plus avisé sur le recyclage littéraire que suppose le PMG. En effet, en soulignant la dimension plagiaire des flyers, ce dispositif collectionneur se contente d'enfoncer une porte ouverte, puisque la composition des cartes se fonde précisément, comme le démontre Liliane Kuczynski, sur la circulation de syntagmes figés :

[...] il existe, en fait, un très petit nombre de modèles de base, reproduits à l'identique d'une carte à l'autre depuis plus d'une quinzaine d'années. Les variantes de ces modèles sont réalisées selon une technique de collage, phrases juxtaposées sans souci de cohérence créant un inventaire hétéroclite, tels les "cadavres exquis » des surréalistes. Mais cette reproduction à l'identique, les marabouts y tiennent fermement, et laissent à leurs imprimeurs très peu de latitude pour redresser telle formule, corriger telle faute: tout se passe souvent comme si ce texte, au-delà du sens, était une sorte de figement ne valant que par sa répétition exacte $^{28}$. 
Figure 11 : Page du site web de la magopinaciophilie proposant de générer son propre flyer de marabout

\section{Personal Marabout Generator}

Saisissez vos informations (sur la gauche, à la place des données d'exemple) pour générer votre flyer. Aucune donnée n'est sauvegardée sur le site. Tous les textes générés aléatoirement sont composés d'extraits authentiques de véritables flyers.

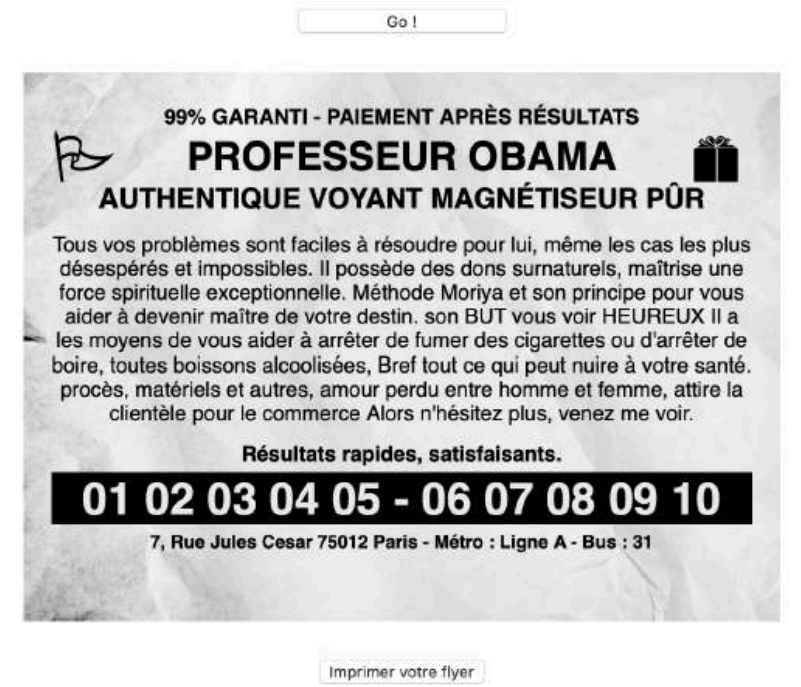

19 La fonction du texte proliférant qui abonde sur les cartes de marabout n'est donc pas à proprement parler informative, mais bien incantatoire. La carte de visite est une carte de redite - et il n'est à ce titre guère surprenant que ses bribes textuelles viennent abreuver des expérimentations poétiques, telles que le recueil d'olivier Cadiot, Retour définitif et durable de l'être aimée ${ }^{29}$. Fidèle à cet intitulé, l'auteur se livre à un jeu de surenchère en prolongeant les énumérations hétéroclites caractéristiques du genre, qu'il se réapproprie de façon plus spectaculaire encore que Sami Tchak et Calixthe Beyala $^{30}$. Sur la quatrième de couverture, le lecteur découvre ainsi une liste de vœux, qui pourrait être lue comme un sommaire de l'ouvrage: "difficulté en amour, explosion du passé dans le présent, traversée du végétal, accès à poussières, possibilités de chansons, amulettes futur». Le projet ainsi ébauché est d'autant plus riche qu'Olivier Cadiot, auteur par ailleurs d'un «art poétic'« inspiré par la grammaire du bon usage, fonde son écriture sur le détournement et le collage de bribes textuelles préexistantes $^{31}$. Le personnage de Robinson, égaré dans l'intarissable flux de paroles d'un dîner mondain, réfugié sur un balcon parisien où il rêve à une existence d'ermite, trouve ainsi dans les fragments des marabouts un refuge et un ferment poétique extrait $\mathrm{du}$ « brouhaha ${ }^{32} »$ contemporain.

La présence littéraire du marabout revêt enfin une signification sensiblement différente chez Patrick Grainville. Après avoir appelé l'écrivain à recouvrer sa " majesté » et à se comporter lui-même en "marabout merveilleux ", maître de son geste et de sa parole, Auguste Traoré lui recommande en effet de procéder à un véritable sacrifice en littérature : il l'invite alors à composer un «conte » de quinze pages, puis, quelle qu'en soit la qualité, à y renoncer en le brûlant et en s'interdisant tout «micmac» et toute récupération ultérieure ${ }^{33}$. La cure passe ainsi par une immolation, qui représente l'exact inverse du geste de recyclage maraboutique mis en 
évidence par Liliane Kuczynski et scelle, par un acte solennel, l'inertie du texte sacrifié. La muse n'est pas loin ici de se muer en « fétiche » attendant ses offrandes.

21 S'il n'a pas l'éclat du dandy noir et préfère l'humilité du colporteur à la chatoyante «marque de l'écrivain ${ }^{34}$ », le marabout n'en demeure donc pas moins un vecteur de contre-littérature largement inexploité dans les textes des auteurs francophones contemporains, enclins à récuser toute identification hâtive avec un charlatan de mauvais aloi. Dans ces conditions, des auteurs comme Olivier Cadiot de Patrick Grainville paraissent plus prompts à emboîter le pas au marabout pour faire de lui un modèle en littérature ou pour trouver chez lui l'indice d'une posture littéraire postcoloniale ${ }^{35}$. Contrairement aux personnages ébauchés par Sami Tchak et Calixthe Beyala, rivés à leur télévision et à leur coin de trottoir respectifs, le marabout se révèle une figure mobile, propice à l'amorce d'une réflexion sur les enjeux d'un recyclage textuel interculturel. La collection du magopinaciophile n'a dès lors pas la fixité morbide de celle du lépidoptériste épinglant ses papillons: si le marabout est un personnage complexe, aussi bien romanesque que poétique, c'est parce qu'il ouvre la perspective non pas d'un "retour définitif et durable » à une hypothétique tradition africaine, mais d'une succession d'allers-retours dans «le grand Mali des mots ${ }^{36}$ ».

\section{NOTES}

1. Patrick Grainville, La Main blessée, Paris, Le Seuil, 2005, p. 281.

2. Voir Jean-Loup Amselle, Branchements: anthropologie de l'universalité des cultures, Paris, Flammarion, 2001.

3. Voir Blaise N'Djehoya et Massaër Diallo, Un regard noir, Paris, Autrement, 1984, p. 127.

4. Souleymane Bachir Diagne et Jean-Loup Amselle, En quête d'Afrique(s): universalisme et pensée décoloniale, Paris, Albin Michel, 2018, p. 59.

5. Le portail de la magopinaciophilie rassemble en ligne près de 2000 prospectus. Voir la page suivante : http://www.megabambou.com/galerie/, consultée le 31 mars 2018.

6. Voir la page suivante: http://www.megabambou.com/encyclopedie/collection/nom.html, consultée le 31 mars 2018.

7. Ibid.

8. Bernard Mouralis, Les Contre-littératures [1975], rééd. Paris, Hermann, 2011, p. 54-55 : «En dehors de cette catégorie relativement facile à repérer - l'ouvrage - parce qu'il est toujours possible d'en donner au moins une définition matérielle, il faut également prendre en considération tout le reste, c'est-à-dire l'ensemble des textes et des signes qui sont produits et transmis en dehors des structures de la culture lettrée du moment et qui sont toujours susceptibles de solliciter notre attention ou notre regard. Domaine immense où le langage écrit se trouve utilisé d'une manière particulière - non "littéraire" [...]. "

9. Ibid., p. 12.

10. Voir à ce sujet Jan Blommaert, Grassroots Literacy: Writing, Identity and Voice in Central Africa, London, Routledge, 2008.

11. Voir Blaise N’Djehoya et Massaër Diallo, Un Regard noir, op. cit.

12. Voir Liliane Kuczynski, Les Marabouts africains à Paris, Paris, CNRS Éditions, 2002. 
13. Ibid., p. 6.

14. Le terme de "bricolage ", tel qu'il est employé par Liliane Kuczynski, est emprunté aux analyses de Lévi-Strauss précisées par André Mary. Voir par exemple André Mary, «Bricolage afro-brésilien et bris-collage post-moderne », dans Philippe Laburthe-Tolra (dir.), Roger Bastide ou le réjouissement de l'abîme, Paris, L'Harmattan, 1994, p. 85-98. On notera que le marabout consulté dans le roman de Patrick Grainville applique d'ailleurs à la lettre ces préceptes syncrétiques lorsqu'il assure à son client qu'il emploiera « des mots de [s]a culture ». Voir Patrick Grainville, La Main blessée, op. cit., p. 281.

15. Liliane Kuczynski, Les Marabouts africains à Paris, op.cit., p. 32. Voir également le passage suivant, p. 26: «Pourtant, si maintes descriptions normatives du personnage du marabout recueillies à Paris, mettent bien l'accent sur le maîtrise de l'écriture "avec les noms de Dieu", "avec les mots et les secrets du Coran", si, disent certains, la relation d'un marabout à son disciple ou à son consultant s'établit par l'intermédiaire de la "tête de la plume" (qui écrit) spécialisation permettant d'opposer le marabout au "féticheur" connaisseur des plantes et des racines, et au "sorcier" bénéfique (biledjo), chargé en particulier de libérer de l'emprise des "mangeurs d'âme" - cet apanage n'épuise en rien la complexité du personnage. »

16. Voir par exemple Justin-Daniel Gandoulou, Au cœur de la Sape : mœurs et aventures des Congolais à Paris, Paris, L'Harmattan, 1989.

17. Voir notamment Alain Mabanckou, Bleu blanc rouge, Paris, Présence Africaine, 1998; Alain Mabanckou, Black Bazar, Paris, Le Seuil, 2009 ; et Alain Mabanckou, Le monde est mon langage, Paris, Grasset, 2016. Voir également Daniel Biyaoula, L'Impasse, Paris, Présence Africaine, 1996 et Julien Mabiala Bissila, Au nom du père, du fils et de J. M. Weston, Paris, Acoria éditions, 2013. Sur le rôle du sapeur dans la littérature francophone contemporaine, voir également Lydie Moudileno, Parades postcoloniales : la fabrication des identités dans le roman congolais, Paris, Karthala, 2006.

18. Voir Black Mic-Mac, 1986, France, 93 minutes. On notera de même que marabout et sapeur constituent deux figures convoquées dans l'imaginaire du chanteur Maître Gims, auteur du tube «Sapé comme jamais » en 2015 mais aussi de « Marabout » en 2017.

19. Voir Thierry Pfister, Le Nègre du palais, Paris, Albin Michel, 1994. Voir aussi à ce sujet Sylvie Jumel, La Sorcellerie au cœur de la République, Chatou, Canot, 2002, p. 153 : « Qui est le "nègre" ? Un personnage que le romancier nomme Anta N'Golo. On ne saura jamais trop de quel pays africain il est issu, mais il est un vague neveu d'un authentique marabout assidument consulté par un président de la République française, président dont le lecteur n'apprendra jamais le nom. Dans quel palais? Bien sûr l'Elysée, jamais nommé non plus. Peu importe, Pfister n'a pas voulu écrire un roman à clés réservé aux intimes du pouvoir. Le président à peine romancé était François Mitterrand. Et Le Nègre du Palais n'est autre que le récit de la sorcellerie ordinaire invité à prendre ses quartiers à l'Elysée par le plus croyant, le plus angoissé, le plus pharaonique et le plus superstitieux des agnostiques, Mitterrand. »

20. Voir Laure Adler, À ce soir, Paris, Gallimard, 2001.

21. Sur cette notion, voir Jacques Chevrier, "Afrique(s)-sur-Seine: autour de la notion de "migritude" ", Notre Librairie, n 155-156, juillet-décembre 2004.

22. Nous pensons ici aux leçons données par Alain Mabanckou à la suite de la son élection à la chaire de création artistique du Collège de France. Voir notamment Baptiste Liger, "Alain Mabanckou, sapé comme jamais ", L'Express, 27/08/2016, [en ligne] et consulté le 15 avril 2018.

23. Sami Tchak, Place des fêtes, Paris, Gallimard, 2000, p. 87.

24. Calixthe Beyala, Comment cuisiner son mari à l'africaine, Paris, Albin Michel, 2000.

25. Voir le portrait peu flatteur du marabout, piètre élégant et mauvais parieur, chez Calixthe Beyala, Comment cuisiner son mari à l'africaine, op. cit., p. $47:$ : - Tu l'aimes, c'est pour ça que tu as amené la poussière de tes pieds chez moi! Quelle belle passe, ouaoh! But! But! J'aurais dû parier, oh merde! Ses yeux sortent de leurs orbites. Un filet de sueur perle à ses tempes 
grisonnantes. Il bondit sur ses pieds et je vois qu'il porte un pantalon rétréci par les lavages imprudents ; cela et aussi ses sandales d'où pointent des orteils durillonnés par des chaussures trop serrées ». On est loin ici de l'élégance d'un dandy noir. La démonstration de la vacuité des promesses maraboutiques est également frappante chez Fabienne Kanor, Je ne suis pas un homme qui pleure, Paris, JC Lattès, 2016, p. 69 [édition électronique] : «Prenez ma tante, celle qui donnait ses francs aux marabouts parce que son mari allait cajoler ailleurs. Ma tante a dû attendre l'âge de la ménopause pour constater les effets positifs de la magie sur son couple. Ce n'est que dixsept ans après avoir ingurgité 100 millilitres du philtre ne me quitte pas que son vagabond s'est mis à présenter des troubles érectiles. Si donc, vous lisez dans un traité de magie "résultats immédiats", comprenez bien que cela peut tout aussi bien signifier demain que dans perpette. » Enfin, dans un journal de bord paru dans Libération, c'est plutôt la cupidité des marabouts que dénonce ironiquement l'auteur. Voir Fabienne Kanor, «Entre deux terres », Libération, 5 avril 2008, [en ligne], consulté le 15 avril 2018: «D'après le professeur Koma, grand marabout aux dons hérités de la pure source de son père, et croisé métro Marx Dormoy, rien n'est perdu, tout est une question de booking : le maître reçoit tous les jours sur rendez-vous. Time will tell mais time is fric, et c'est à grandes enjambées que j'échappe à ma destinée pour récupérer la ligne 4. »

26. Liliane Kuczynski renvoie à cet égard au jugement de Paul Marty et Maurice Delafosse. Voir à ce sujet Liliane Kuczynski, Les Marabouts africains à Paris, op. cit., p. 22 : «Pour Marty et Delafosse, les marabouts sont les preuves vivantes de la corruption de l'islam avec le "fétichisme ambiant", certaines pratiques, communes aux sorciers et aux marabouts, tournant souvent, écrit Marty, au "charlatanisme" [...]; il donne plusieurs exemples de marabouts vagabonds, quêteurs et magiciens qui furent condamnés par les autorités coloniales pour "maraboutages" (terme extrêmement dépréciatif) ou "pratiques frauduleuses". » Voir Paul Marty, Études sur l'islam et les tribus du Soudan, 4 tomes, Paris, Ernest Leroux, 1920, 4 vol., et Maurice Delafosse, Haut-Sénégal Niger, t. III, Les Civilisations, Paris, Maisonneuve et Larose, 1912.

27. Voir la page «Le monde des flyers de marabouts» du portail de la magopinaciophilie, consultée le 31 mars 2018, [en ligne].

28. Liliane Kuczynski, Les Marabouts africains à Paris, op. cit., p. 357.

29. Olivier Cadiot, Retour définitif et durable de l'être aimé, Paris, POL, 2002.

30. Dans un registre similaire, on citera également la série d'œuvres que l'artiste allemande Friederike Klotz intitule Voyance.

31. Voir Olivier Cadiot, L'Art poétic', Paris, POL, 1988. Voir également à ce sujet François Cusset, "politique de Cadiot ", Vacarme, vol. 40, n 3, 2007, p. 78-79. " Déjà, Retour définitif et durable de l'être aimé - à commencer par ce titre en forme de promesse de marabout au fond d'une boite aux lettres - crépitait de chansons Nike ou Prada et de solos jazz plus ou moins contrôlés (“ouin'mor'taï-m"'), grouillait de personnages typés jusqu'à l'atypique [...] et de lambeaux de Jours de France ou du magazine Détective. Un flux dont le "je" narrateur semblait le témoin dépassé, la victime désignée [...] incapable d'arrêter en lui la machine propositionnelle, et autour de lui le vrombissement du Zeitgeist. Ainsi que Cadiot l'a lui-même suggéré, tout se passe comme si le poète devait être atteint d'un étrange syndrome de Stockholm, envahi par les voix, zébré de mots d'ordre, bombardé de mots de passe, “otage passé à l'ennemi, conquis par les paroles des autres, ffft, avalé, disparu". »

32. Nous empruntons cette expression à Lionel Ruffel, Brouhaha: les mondes du contemporain, Lagrasse, Verdier, 2016.

33. Voir Patrick Grainville, La Main blessée, op.cit., p. 282-283 : «- Mais si c'est mon plus beau texte ? - C'est justement à lui que tu dois renoncer pour que sa beauté remonte dans tout ton être. Son esprit même. - Mais je vais me souvenir de mon texte, je peux toujours tricher, le retrouver, le maquiller, le transposer... - Tu t'interdis ce micmac. Tu as lâché tes mots, tu as ouvert ta main. Tu es libre... Tu oublies le texte. C'est parce que tu aimes ce texte que tu lui rends 
sa liberté. [...] Par amour, tu as ouvert ta main. Tu chevauches en liberté. Le grand Mali des mots s'ouvre à toi, à perte de vue.»

34. Voir Marie-Ève Thérenty et Adeline Wrona, L'Écrivain comme marque, Paris, Presses de l'université Paris-Sorbonne, à paraître.

35. Voir à ce sujet Jérôme Meizoz, Postures littéraires, mises en scène modernes de l'auteur, Genève, Slatkine, 2007, et Anthony Mangeon (dir.), Postures postcoloniales. Domaines africains et antillais, Paris, Karthala, 2012.

36. Patrick Grainville, La Main blessée, op. cit., p. 283.

\section{RÉSUMÉS}

Cet article entend se pencher sur le terrain congru que représente un pas de porte investi par les prospectus des marabouts. Nourrissant une véritable pulsion collectionneuse dont le meilleur exemple demeure le site de la magopinaciophilie en ligne depuis 2005 , ces cartes de visite sont à la fois l'indice d'un "branchement " interculturel et le support d'une potentielle circulation poétique. L'examen des occurrences littéraires du marabout africain, dont on cherchera ici la trace chez Sami Tchak, Calixthe Beyala, Olivier Cadiot et Patrick Grainville, témoigne cependant d'une relative discrétion de cette figure, aujourd'hui largement occultée par la silhouette chatoyante du sapeur.

The present article reflects on a very narrow fieldwork: by focusing on a mailbox overwhelmed by the promotional flyers edited by Parisian marabouts, I intend to investigate the abundant literary follow-ups of frequently dismissed textual objects. Those flyers, which have been collected and indexed on-line since 2005, allow for an intercultural connection between Africa and Europe as well as for the circulation of poetical fragments. Although marabouts distinctively appears in works by Sami Tchak, Calixthe Beyala, Olivier Cadiot and Patrick Grainville, there presence remains discrete and isolated and they are to this day overshadowed by the prominent literary figure embodied by the "sapeur".

\section{INDEX}

Mots-clés : Marabouts, Collection, Littérature francophone, Circulations textuelles

\section{AUTEUR}

\section{NINON CHAVOZ}

NINON CHAVOZ, actuellement ATER à l'Université Paris 3-Sorbonne Nouvelle, est l'auteur d'une thèse intitulée « La tentation encyclopédique dans l'espace francophone africain : des documentations coloniales aux glossaires contemporains ", soutenue en 2018 sous la direction de Xavier Garnier. 